\title{
Monoclonal Antibody R24
}

National Cancer Institute

\section{Source}

National Cancer Institute. Monoclonal Antibody R24. NCI Thesaurus. Code C2349.

An IgG murine monoclonal antibody directed against the gang lioside GD3 glycolipid, located in the cell membranes of some tumor cells. Monoclonal antibody R24 binds to GD3-positive cells, thereby initiating antibody-dependent cytotoxicity against GD3positive cells. ( $\mathrm{NCl04)}$ 